\title{
Design Health care system using Raspberry Pi and ESP32
}

\author{
Qunoot N. Alsahi \\ Computer Science Dept. \\ Collage of Education for Pure Sciences \\ University of Basrah
}

\author{
Ali F. Marhoon \\ Electrical Engineering Dept. \\ Collage of Engineering \\ University of Basrah
}

\begin{abstract}
The focus of this paper is to implement an intelligent health care system based on internet of thing (IOT) for the measurement of the vital signs like pulse rate, temperature, spo2, ECG, using (ESP32 div kit v1) for wireless wearable sensor controller and raspberry pi 3 as a server. With the system proposed, the doctor can save work time to visit the patients that responsible about them and any facilitates monitoring the huge number of patients. The WI-FI technology is utilized as a communication tool to allow transmission the data remotely. The data of patient are sent to the web server to be stored in the database and view the data on the web page anywhere and anytime using smart devices and alert the doctor to any abnormal state. This work with the intelligent health care system provide an efficient medical service ,by collecting and recording the informant that include heart rate, ECG, temperature and spo2 that enable the doctor to monitor his patient with flexibility and confidence .
\end{abstract}

\section{Keywords}

Healthcare, IOT, raspberry pi, ESP32, ECG, MQTT, WSN, medical wearable electronic, AD8232, patient monitoring, Node-Red.

\section{INTRODUCTION}

Nowadays, in most countries the death is increasingly because health problem day by a day. Each year the rate of death is 55.3 million of people ,this rate due to a delay in giving proper treatment or lack of care due to lack of medical staff or resource especially in rural hospital. Hence, there is a need for some techniques to overcome this problem. This paper proposed a design of an intelligent healthcare monitoring system based on IOT [1], [2].

Internet of thing (IOT): is a concept of network that is connected several devices (portable, wearable, house - held device etc...), network internally, also combine software with hardware in network, and connected this with other network. IOT environment allow supervising control or transferring remotely from one network to another one in that infrastructure. In the healthcare field, IOT helps to real-time monitoring system with more flexibility and important vital function of the people in any time and place. Therefore, it can transfer the data acquired remotely in real-time, which ensure real-time monitoring to patient status [1], [3], [4].

The pervasive monitoring system that able to transfer a patient's vital sign to medical application renewal in real-time. The two mainly parts of the system is the data collection part and the transmission part .The public architecture of the application based on IOT has three layers, the first layer is The sensing, The second is the transport and the third is the application. This type of architecture is evident for most surveillance systems [2].
Many project and research have been introduced are focused on a real-time and health patient's condition monitoring in a smart way and introduce healthcare medical service remotely.

In 2015, Ali.F and Ali.H [5], developed long term monitoring system that detects the abnormal condition of the elderly at home and performs all daily activities using Shammir Kit, which is considered costly in terms of price.

In 2016, Ganesh et al [6], had design a smart chair to provides the medical parameters, but it is not suitable to track the patient's condition due it impedes the patient's movement and execution of daily activities. But in 2016 Gupta et al [7], proposed system using board (INTEL GALILO 2ND) to provide the medical services and adopt temperature and heart rate information in diagnosing the health condition, but the ECG, which is one of the most common chronic diseases that affects the elderly, has not been adopted.

In 2017, Chao et al [2], proposed system to monitoring the physiological behavior of the patient in four modes of data transmission to the healthcare center to achieve the required balance of communication and computing resources. Hence, the type of data transmission determined by the severity of the disease, Hence, the mode selected is suitable for patient state , that's where the mode is either real - time monitoring that require continues follow up or the monitoring that adopt comparison to some vital measurement normal with data collected from sensors layer. As these two types of level of serious cases of patients while the other two types are the lowest level of risk suffered by the patient. In the mode of data transmission in the lowest level of risk on patient state, it is in specific periods in form either continuously or in case of demand In accordance with the criteria specified by the competent doctor. In this system, adopt two communication techniques that is Bluetooth for short area and the Wi-Fi for the remote application.

In 2017, Melisa and Nagapriya [8], proposed a system that using (LPc212 Arm Processor) Development Board to design health-monitoring system to measure the pulse rate and body fat, etc. This system can Introduce medical care service in efficient way and facilitate the doctor work to get information about his Patient. It can send this information to server and make the doctor able to access these data by web page. In addition, the system can detect the patient and decisionmaking about the patient status.

In 2017, pardeshi et al [9], proposed health monitoring system for get many measurement of all the body such as (pressure, temperature, ECG signal) of human. The system capable to capture any abnormality value of the measurement that a quire from the sensing platform that connected to the raspberry pi and alert the doctor or expert via GSM or WIFI technology, This modern system save patient from the advance health 
problems.

In 2017, Divakaran et al [10] had design system that used many type of medical sensor to build diagnosis system to collect data and exchange data with central station of monitoring for interpretation. The system uses STM32F429 microcontroller as a web server and ROST as an operating system to handle request data. The goal of this system is provide vital Parameter and live video image to display Patient status to the medical Expert to help in health status of his patient by logged in Page. This page consist of 3 tab which is one for display The video and two other one for the vital parameter and the other for display report of the patient status.

In 2017, Uddin et al [11], proposed system design for monitoring in intensive care unit (ICU) to make the specialists monitor what is happening to patient in ICU continuously and receive notifications of emergency cases by used various sensor attached in Arduino 101 to gather the information and send it to cloud to understand it according to determined equation. This monitoring system makes the Physician capable to monitor more than one Patient in ICU in real time.

But in the system was proposed in 2018, Evian and et al [4] have adopted AWS and Mangoose OS in designing a system to transmit data and send an alert to the doctor without storing data and refer to it at a later time for specific reasons. While Stradolini et al [12], use smart watch for stay live with critical patient status. Hence, the doctor that responsible for unit can access the information and receiving notification by his smart watch.

The system contains three parts:

i. Tablet running on android platform sit client side.

ii. Tablet for server side.

iii. Smart - watch running application sit final side is the doctor.

In this paper, it proposed an intelligent health monitoring system based on IOT platform. This system monitor and follow up the patient's health condition intelligently, which it can measure the vital signs such as (temperature, ECG, SPO2). So implemented the system to provide medical care for patient in real-time and give alert to control disease before it worsens.

\section{MATERIALS AND METHOD}

This work aims to design health care system based on IOT to collected multi parameter of patient body and analysis to detect any abnormality value and sent it to Server to be stored and Email the physician to get the information about patient health status and display the parameter that measured in web page.

Several type of medical sensor is utilized to gathers the patient vital parameters and transfer to the central monitoring station for view to the doctors. In a simplest term, the proposed system consists of three major subsystems as shown in the following

1- Data collection subsystem that represented by the sensing layer.

2- Wireless communication subsystem that represented by the transport layer.

3- Data visualization and storing subsystem that represented by the user interface.
The main components of the proposed healthcare system are illustrated in Fig.1. which comprises Esp32, Raspberry Pi 3 and MQTT protocols. The node MCU allow many peripherals to connect with it, in this work the peripherals represent several sensors such as ECG/Pulse ate, SPO2 and temperature.

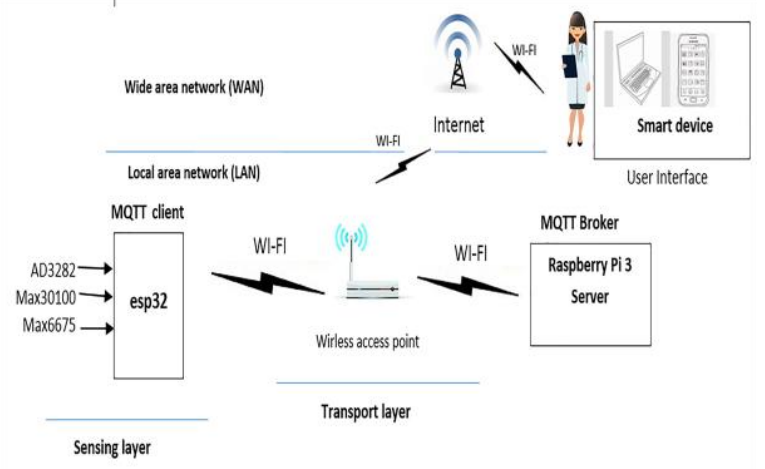

Fig 1: the overall system architecture

\subsection{Data Collection Sub System}

Many type of medical parameter can gather using various medical sensors such as pulse rate, body temperature, heart response, blood pressure, respiratory and Capillary oxygen saturation etc. The analog data that acquire from the sensor must processed and convert to digital format to fit the storage and transmission purposes. The proposed model in this paper employs the following sensors:

- $\quad \mathrm{AD} 8232$ for 3-chanal ECG monitoring.

- MaX30100 for spo2 measurement.

- Max6657 for temperature measurement.

\subsubsection{Heart Respond Measurement}

The electrocardiogram is the metric to electrical heart activity, it represent as an analog signal contain P,Q,R,S,T waves . The heart's job is to pump blood in two stages, which is systolic and diastolic, this two stages form electrical heart activity. Heart rate and regularity can be measured by ECG, as well as offer indirect way to prove influx blood to the muscle of the heart. When measure the ECG signal, it need to direct connection with the patient's skin, which it is set the electrodes on the body, and used different methods to obtain the ECG signal. So it is used the AD8232 3-chanell ECG sensor that is high accuracy and cost-effective solution to obtain ECG data [4],[10].AD8323 have three electrode was connected to right Aram(RA), left Aram (LA) and right leg (RL) respectively, [13], [9] .fig.2. Illustrate the ECG sensor and probe Placement

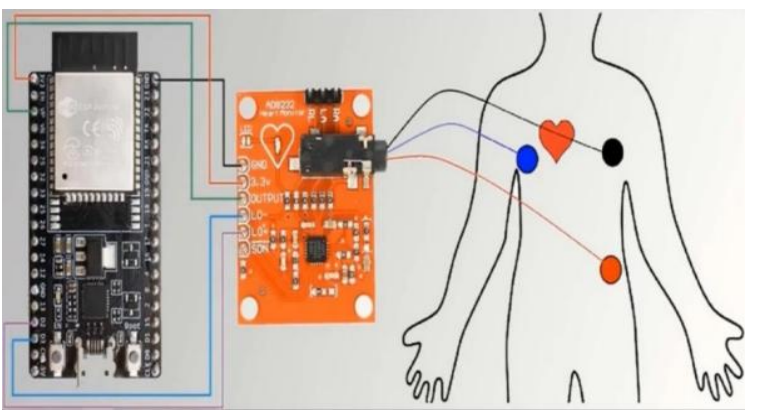

Fig 2: ECG sensor with ESP32 connection and probe Placement of ECG electrode 


\subsubsection{Blood Oxygenation Monitoring:}

Oxygen saturation (spo2) is a measurement of how much blood oxygen is carrying as a percentage of the maximum it could carry [10]. The oxygen saturation measured by max 30100 that illustrate in fig. 3 . The MAX30100 is the solution for integrate heart rate with pulse oximetry measurement sensor. The sensor designed to be suitable with the wearable techniques. The working principle of the sensor is optical reflection, so it is fitted with two LED, which is a photodetector and optimized optics, in additional that, it perform processing for low noise analog data to obtain good outputs for pulse rate and SPO2.The sensor placed on a fingertip for data acquire. The data transfer between Max 30100 sensor and Esp32 is accomplished by using I2C communication protocol via SDA and SCL pins of both as shown in Fig 3.

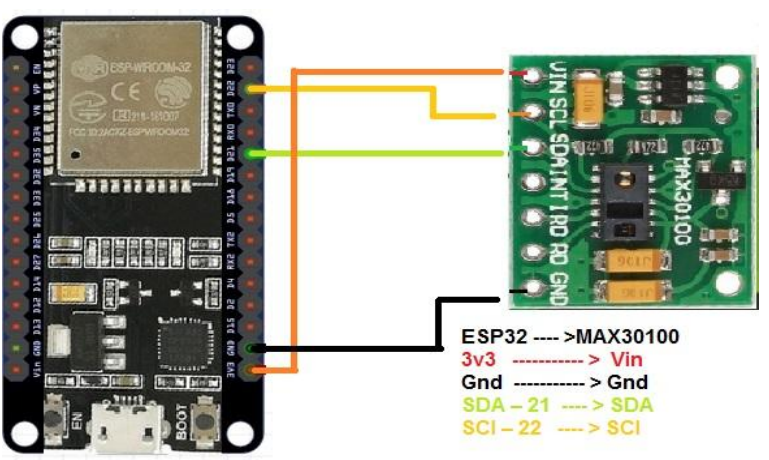

Fig 3: interfacing between Max 30100 and esp32

\subsubsection{Body Temperature Measurement:}

Body temperature is a metric of heat emitted from the human's body there are four way to take a temperature:
1- Axillary method.
2- Oral method.
3- Tympanic method.
4- Rectal method.

Temperature is measure by max6675 sensor that display in fig.4 that used any of the previous way to get the body temperature parameters. To configure the sensor with esp32 using SPI communication protocol, as shown fig. 4 .

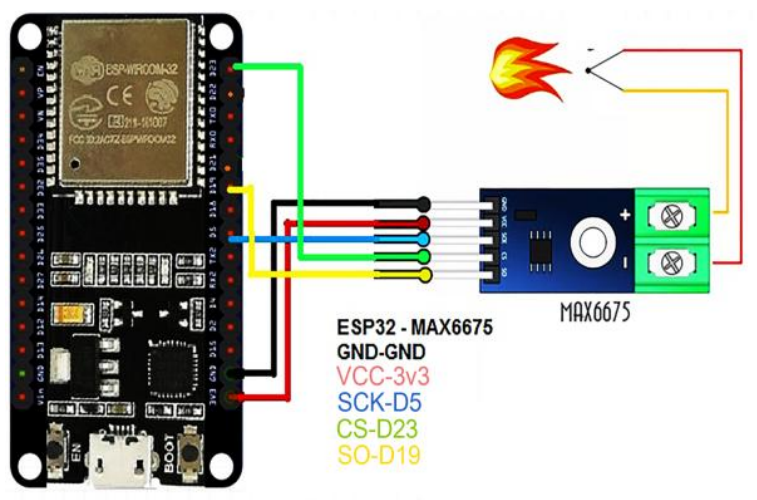

Fig 4: Configuration of temperature sensor with ESP32

\subsubsection{Data Acquisition With NodeMCU (ESP32):}

ESP32 is a microcontroller designed to handle with low power and low cost system, and it has the possibility and capabilities of Arduino, as it can be dealt with programmatically through the Arduino IDE and Micro-python environment. Esp32 is a high performance 32-bit LX6 microprocessor, ultra-low power co-processor 3.3VDC. It has 6-chanel 12-bit input ADC pins, 2-chanel output DAC pins, 3URTs, 2-SPIs, 3-I2C, flash memory 4MB, in addition to 520KB SRAM [9],[4]. Therefore, it is suitable for configuration of the sensor that used in our system. The data transfer, data acquire data analysis and diagnosis accomplished in ESP32.

\subsection{Communication Sub System}

Communication functionality is the one of the main objectives of this work that is to enable connected fast and low cost. The core communication subsystem is used ESP32 due provide two way of communication (Bluetooth and WI-FI) and require simple configuration with the network, as well as it was low cost, small size and highly performance for processing. Microcontroller functionality Esp32 is performs data processing and transmission. The MQTT protocol is utilized as a transport protocol to perform the task of transferring data to the server via Wi-Fi chip that are configured with the IOT devices.

\subsubsection{Message Queuing Telemetry Transport (MQTT):}

MQTT is lightweight message protocol, which resides at the top of TCP/IP protocol that is illustrated in Fig.5. MQTT is an event driven Publish /subscribe architecture. In a publish and subscribe system, a device can publish a message that contain the information to be exchange between devices on a topic that specify where publish the message, or it can be subscribed to a particular topic to receive messages. MQTT Broker drives the whole communication. MQTT broker is responsible for receiving and sending the message among the sender's devices and the correct receiver's devices. Therefore, MQTT is a perfect communication protocol for IOT application. The MQTT have multiple task, such as read and publish sensor's parameters, as well as, control of the output objects by sending a commands, consequently, it is very easy to start communication with many IOT devices [9], [14].

\subsubsection{ESP32 with MQTT:}

ESP32 Configured to connect to Wi-Fi network and saves the information of the SSID and Password to use it in the connection of the network. Once ESP32 connect to the network, it will works as a MQTT client to publish data on the MQTT topics in the MQTT broker[14], where MQTT broker running on a raspberry pi as illustrated in fig.5.

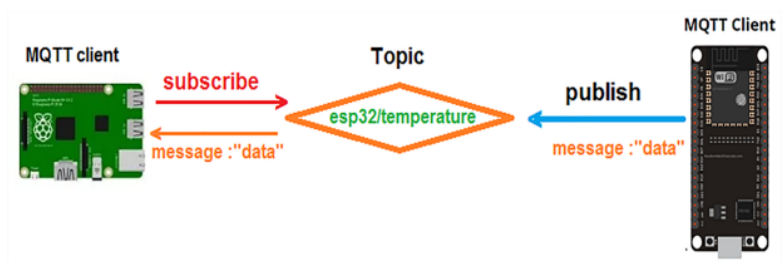

Fig 5: data publishing process

\subsubsection{Raspberry Pi as MQTT Broker:}

Raspberry Pi is a small and low cost computer, and also it is a powerful platform to interfacing with several devices. The running of Raspberry Pi somewhat similar to standard PC, so it is need display unit, mouse, keyboard, Power supply, and micro SD card work as a hard disc in Pc. The raspberry Pi, as any device, requires operating system to work. It run 
Raspbian OS that based on Linux. To enable Raspberry Pi as a server, need install MQTT Broker and enable it to allow auto-start. There are several brokers can use. In our healthcare monitoring projects use the Mosquitto broker, which is well support on Debian based Linux platforms. As well as need to install MySQL, Apache and PHP [4],[13],[15].Raspberry pi is a main server, which save history of the patient health and provide the information to the end point, as well as it run as MQTT client to enable publish/subscribes to the Topics as illustrate in fig.6.

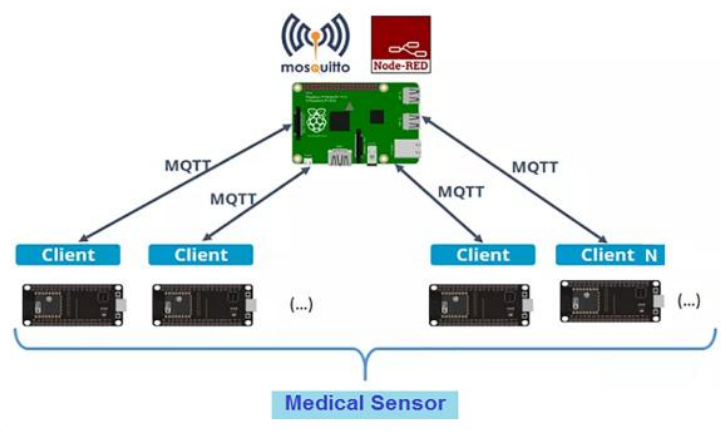

Fig 6: MQTT broker with Raspberry pi

\subsection{Data Visualization Sub System}

In order to view all the patient's vital signals in an easy way, a Node-RED tool is utilized. In addition to that, and to manipulate the database of the patient's information, a web application is designed to facilitate the tasks of different system users.

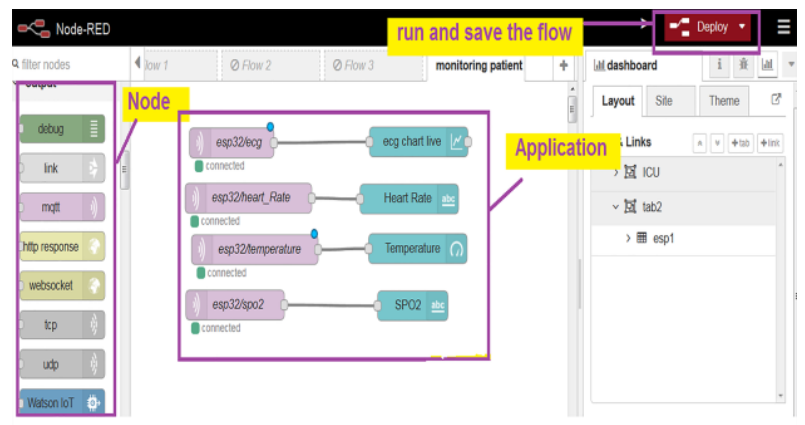

Fig 7: Node-RED interface

Node-RED is a powerful programming tool developed to visualize many of IOT Applications. It runs with several types of software and hardware, such as Android, Arduino, AWS, Raspberry Pi, etc. Generally running the tool by executing of the command line (sudo node-red-start) in the raspberry pi terminal to start the service of Node-RED and displays its log output [16]. On the other hand, one can access the Node-Red tools from any external device, by typing the IP address of the Raspberry pi followed by (1880), default TCP port for NodeRED, (http://192.168.0.190:1880) in browser to display the Node-RED interface. Fig.7 explains the overall interface of the Node-RED tool includes the application node for monitoring patient vital signs.

\section{RESULT AND DISCUSSION}

In this work, MQTT protocol is adopt for data transfer process due it is lightweight protocol and using effectively the network constrains like high latency and bandwidth. So, when the sensor nodes need to be expanded a small code is required.
The architecture of MQTT is publish/subscribe adopt being message oriented information to exchange the data, therefore, it is offer wide conjunction to serve dynamic environment of the systems, which it require handle enormous amount of the messages and objects. Client of MQTT able to handle more than 10,000 clients. As well as MQTT offer guarantees of message delivery services such as (QOS) [9],[17]. In additional that, it an easy communication tool for application developers to adopt it as a communication protocol [13], [15].Fig.8 illustrated the overall publish/subscribe architecture of the Real-Time monitoring system.

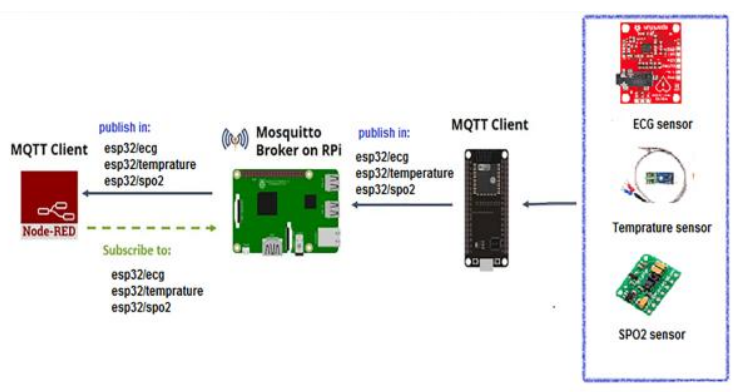

Fig 8: publish/subscribe architecture of the Overall system

The ESP32 publishes ECG data on the esp32/ecg topic, temperature on the esp32/temperature topic and the SPO2 on the esp32/spo2 topic. The Node-RED application subscribed to those topics. So, it receives ECG data, temperature and SPO2 measurement that can be displayed on a chart or gauge. Data acquisition from the Patient are processed locally (inside the ESP32) to detect any abnormal condition in the patient health. In such cases, the ECG pattern signals are transferred to the server to be stored in the database, then generate alert to the doctor, and display these information on the monitor. In order to access the proposed monitoring system from anywhere, one can enter the IP address of the hosting server. Then according to the login information that is entered by the user, the roll of user is specified as (Administrator, Doctor, or Patient), and hence one of the pages are displayed based on the user type. The doctor's page include number of button particular to execute special task, for example display all the patients in charge ,search for patient and can monitor health status as showing in the fig.9. Figure.10 explain the task of monitoring button that enable the user to activate the real-time monitoring for patient in ICU.

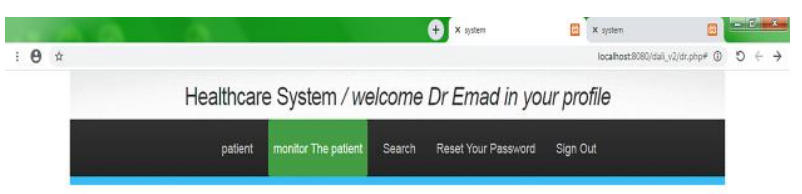

Searching For Patient

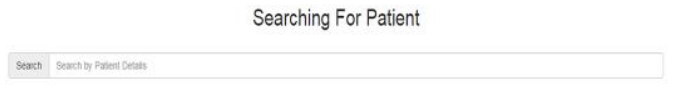




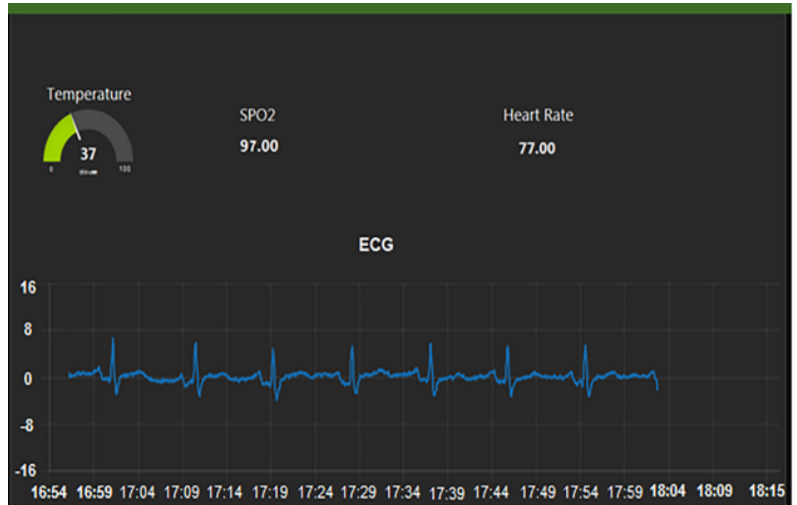

Fig 10: Web application of Real-Time monitoring in healthcare system

The patient page consists of a set of fields that are manually fill up by pathological analyzes measurements (blood glucose, blood pressure and any parameter can measured in the home). The Page of administrator, include some buttons for add user and patients to the system and able to view the patient in two method. The first one is to show a table that contains all

patients enrolled in the hospital database with the possibility to view, search, delete, modify. The other way is to display a list of the patient that followed by the doctor. Figure 11 demonstrate the page of the administrator with the form of user insertion to the system.

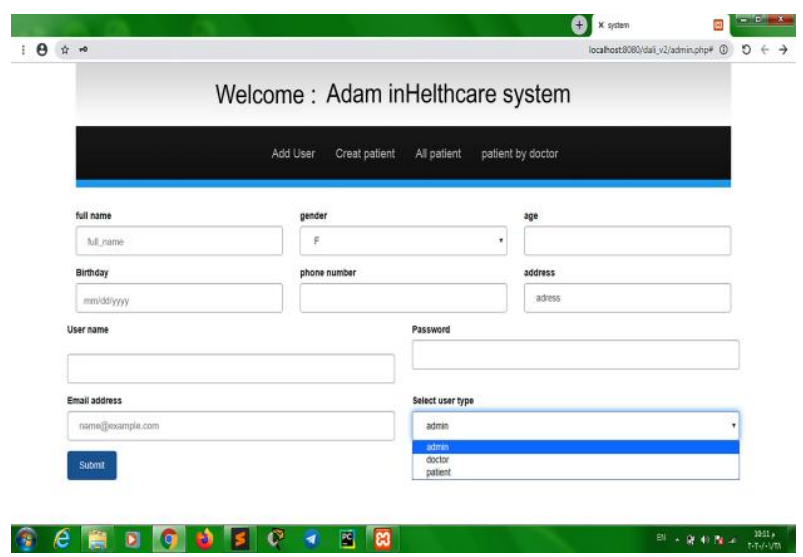

Fig 11: Interface of admin's page

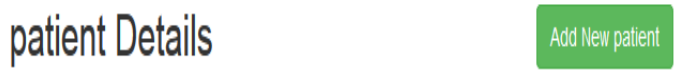

\begin{tabular}{|c|c|c|c|c|c|c|c|}
\hline ID & Name & Address & Email & phone & Dr.name & Age & Action \\
\hline 1 & $\begin{array}{l}\text { shighaf } \\
\text { mustafa }\end{array}$ & basra & shighaf@gmail.com & 07705751001 & firas & 25 & $\begin{array}{l}0 / \\
0 \\
\mathbb{1}\end{array}$ \\
\hline 2 & $\begin{array}{l}\text { qunoot } \\
\text { alsahi }\end{array}$ & iraq & qunootalsahi@gmail.com & 07712566534 & mohammed & 25 & $\begin{array}{l}01 \\
\text { 晋 }\end{array}$ \\
\hline
\end{tabular}

Fig 12: Table of patient in admin's page

\section{CONCLUSION}

The work is an upgraded version of several healthcare monitoring systems. It proposed the use of IOT solution for patient monitoring system, which enable transfer data in fast and secure way. The IOT integrates all components in one system , this make it One Comfortable for the doctor and patient to save time especially living in place far from health centers such as countryside and difficult to frequent hospital visits, another side faster diagnosis and give them the necessary health Service in an emergency.

The system built using an esp32 and raspberry pi make it has reasonable efficiency and very low cost, due low MQTT bandwidth and also low power consumption, make it convenient implement for limited resource in both rural and small health centers.

\section{REFERENCES}

[1] Al-Fuqaha, Ala, et al. "Internet of things: A survey on enabling technologies, protocols, and applications." IEEE communications surveys \& tutorials 17.4 (2015): 2347 2376 .

[2] Li, Chao, Xiangpei Hu, and Lili Zhang. "The IoT-based heart disease monitoring system for pervasive healthcare service." Procedia computer science 112 (2017): 23282334.

[3] Lavanya, S., G. Lavanya, and J. Divyabharathi. "Remote prescription and I-Home healthcare based on IoT." 2017 International Conference on Innovations in Green Energy and Healthcare Technologies (IGEHT). IEEE, 2017.

[4] Medvediev, Ivan, et al. "IoT solutions for health monitoring: analysis and case study." 2018 IEEE 9th International Conference on Dependable Systems, Services and Technologies (DESSERT). IEEE, 2018.

[5] Marhoon, Ali Fadel, and Ali Hussein Hamad. "A new real-time resource-efficient algorithm for ECG denoising, feature extraction and classification-based wearable sensor network." International Journal of Biomedical Engineering and Technology 18.2 (2015): 103-114.

[6] Ganesh, G. R. D., et al. "Design of a low cost smart chair for telemedicine and IoT based health monitoring: An open source technology to facilitate better healthcare." 2016 11th International Conference on Industrial and Information Systems (ICIIS). IEEE, 2016.

[7] Gupta, Punit, et al. "IoT based smart healthcare kit." 2016 International Conference on Computational Techniques in Information and Communication Technologies (ICCTICT). IEEE, 2016.

[8] Pereira, Melisa, and Kamath K. Nagapriya. "A novel IoT based health monitoring system using LPC2129." 2017 2nd IEEE International Conference on Recent Trends in Electronics, Information \& Communication Technology (RTEICT). IEEE, 2017.

[9] Pardeshi, Vivek, et al. "Health monitoring systems using IoT and Raspberry Pi-a review." 2017 International Conference on Innovative Mechanisms for Industry Applications (ICIMIA). IEEE, 2017.

[10] Divakaran, Sindu, et al. "IOT clinic-Internet based patient monitoring and diagnosis system." 2017 IEEE International Conference on Power, Control, Signals and Instrumentation Engineering (ICPCSI). IEEE, 2017.

[11] Uddin, Mohammad Salah, Jannat Binta Alam, and Suraiya Banu. "Real time patient monitoring system based on Internet of Things." 2017 4th International 
Conference on Advances in Electrical Engineering (ICAEE). IEEE, 2017.

[12] Stradolini, Francesca, et al. "Live demonstration: An IoT smartwatch-based system for intensive care monitoring." 2017 IEEE Biomedical Circuits and Systems Conference (BioCAS). IEEE, 2017.

[13] Zilani, Kazi Abu, et al. "R 3 HMS, An IoT Based Approach for Patient Health Monitoring." 2018 International Conference on Computer, Communication, Chemical, Material and Electronic Engineering (IC4ME2). IEEE, 2018.

[14] Yi, Ding, et al. "Design and implementation of mobile health monitoring system based on MQTT protocol." 2016 IEEE Advanced Information
Management, Communicates, Electronic and Automation Control Conference (IMCEC). IEEE, 2016.

[15] Gardašević, Gordana, et al. "A heterogeneous IoT-based architecture for remote monitoring of physiological and environmental parameters." International Conference on IoT Technologies for HealthCare. Springer, Cham, 2017.

[16] Lekić, Milica, and Gordana Gardašević. "IoT sensor integration to Node-RED platform." 2018 17th International Symposium INFOTEH-JAHORINA (INFOTEH). IEEE, 2018.

[17] Komkrit Chooruang, K., and P. Mangkalakeeree. "Wireless Heart Rate Monitoring System Using MQTT." Procedia Computer Science 86 (2016): 160163. 